\title{
Antibiotics do not reduce toothache caused by irreversible pulpitis
}

\author{
Are systemic antibiotics effective in providing pain relief in people who have \\ irreversible pulpitis?
}

\begin{abstract}
Keenan JV, Farman AG, Fedorowicz Z, Newton JT. Antibiotic use for irreversible pulpitis. Cochrane Database of Systematic Reviews 2005, Issue 2. Art. No.: CD004969
\end{abstract}

Data sources Cochrane Oral Health Group Trials Register and the Pain, Palliative Care and Supportive Care Group Trials Register, the Cochrane Central Register of Controlled Trials, Medline and Embase.

Study selection Randomised controlled trials (RCT) were included that involved patients over the age of 18 years who had presented with a single tooth with a clinical diagnosis of irreversible pulpitis.

Data extraction and synthesis Abstracts of studies were independently assessed by two reviewers. The primary outcome was patientreported pain (intensity/duration) and pain relief measured on a categorical scale in the preoperative phase of irreversible pulpitis. Secondary outcomes were type, dose and frequency of medication required for pain relief. Only one trial was included so pooling of data from studies was not possible and a descriptive summary is presented. Results One trial of 40 participants was included. There was a close parallel distribution of the pain ratings in both the intervention and placebo groups over the 7-day study period. The between-group differences in sum pain intensity differences for the penicillin group were $6.0 \pm 10.5$, and for placebo $6.0 \pm 9.5 \quad(P=0.776$; differences assessed by Mann-Whitney-Wilcoxon test considered to be statistically significant at $P=0.05$ ). The sum pain percussion intensity differences for the penicillin group were $3.5 \pm 7.5$ and placebo $2.0 \pm 7.0(P=0.290)$. There was no significant difference in the mean total number of ibuprofen tablets $(P=0.839)$ and acetaminophen tablets $(P=0.325)$ taken for pain relief in either group over the study period. The administration of penicillin over placebo did not appear to significantly reduce the quantity of analgesic medication taken $(P=0.05)$ for irreversible pulpitis.

Conclusions This review based on one methodologically sound but low-powered small sample trial, provides some evidence that there is no significant difference in pain relief between individuals who had untreated irreversible pulpitis who did or did not take antibiotics in addition to analgesics.

\section{Commentary}

The clinical question asked in this review is an important one. Intuitively, the answer is "no", since irreversible pulpitis is not an infection. Nevertheless, numerous studies have shown that there is widespread inappropriate use of antibiotics: up to $74 \%$ of people who have pulpitis are given a prescription for antibiotics. ${ }^{1}$

This report, an excellent example of a high quality rigorous systematic review ${ }^{2}$ examined early stage pulpal disease and found that systemic antibiotics are not effective in relieving pain in these patients. Although only one RCT was located, despite a comprehensive search strategy, the review concurs with the results of two metaanalyses which studied various interventions in acute apical periodontitis $^{3}$ and acute apical abscess, ${ }^{4}$ both of which concluded that there is no evidence to support the use of prophylactic antibiotics in the management of localised endodontic disease in healthy patients.

Address for correspondence: Emma Tavender, Review Group Co-ordinator, Cochrane Oral Health Group, University Dental Hospital of Manchester, Higher Cambridge Street, Manchester M15 6FH, UK. E-mail: emma.tavender@man.ac.uk
With the increase in multidrug-resistant infections such as methicillin-resistant staphylococcus aureus (MRSA), a major and very serious nosocomial pathogen which is also emerging in the community, most, if not all, dentists would disapprove of inappropriate use of antibiotics in the abstract situation. While antibiotics are appropriate for patients with serious systemic infection, when confronted with a suffering patient and armed with personal or anecdotal experience of the rare patient whose condition has progressed from pulpitis to serious systemic infection, many clinicians are tempted to prescribe antibiotics despite the evidence of ineffectiveness, believing that this will provide more benefit than harm for their individual patient.

This erroneous belief can be disastrous. Adverse drug reactions (ADR) to antibiotics are not uncommon, ranging from nuisance side effects to severely debilitating and fatal outcomes, such as toxic epidermal necrolysis (TEN). ${ }^{5}$ In our hospital-based practice, we have been asked to consult on two patients in the past few years who developed near-fatal TEN, but recovered with severe sequelae. Both had been treated with amoxicillin for localised endodontic problems.

The fact that severe ADR are not uppermost in the minds of many clinicians when prescribing drugs can be attributed to a number of factors. Clinical trial designs in dentistry usually exclude people, including the elderly, who have comorbid conditions and would be more likely to develop significant ADR. Many clinical trials have inadequate power to detect uncommon but serious adverse events and meta-analysis primarily examines treatment efficacy, not safety. ${ }^{6}$ Voluntary reporting of ADR to government programmes or pharmaceutical companies is inadequate on a global scale, with reporting rates as low as $4-10 \%$ for life-threatening ADR such as TEN. ${ }^{7}$

\section{Practice points}

- There is no evidence to support the use of prophylactic antibiotics in the management of localised endodontic disease in healthy patients. - Adverse drug reactions, while under-reported, are very common and can be life threatening.

\section{Susan Sutherland}

Department of Dentistry, Sunnybrook and Women's College Health Sciences Centre, Toronto, Ontario, Canada

1. Dailey YM, Martin MV. Are antibiotics being used appropriately for emergency dental treatment? Br Dent J 2001; 191:391-393.

2. Cook D, Sackett D, Spitzer W. Methodologic guidelines for systematic reviews of randomized controlled trials in health care from the Potsdam Consultation on meta-analysis. J Clin Epidemiol 1995; 48:167-171.

3. Sutherland SE, Matthews DC. Emergency management of acute apical periodontitis in the permanent dentition: a systematic review of the literature. J Can Dent Assoc 2003; 69:160a-160l.

4. Matthews DC, Sutherland SE, Basrani B. Emergency management of acute apical abscess: a systematic review. J Can Dent Assoc 2003; 69:660a-660i.

5. Roujeau JC, Kelly JP, Naldi L, et al. Medication use and the risk of Stevens-Johnson syndrome or toxic epidermal necrolysis. N Engl J Med 1995; 333:1600-1607.

6. Mittmann N, Liu BA, Knowles SR, Shear NH. Meta-analysis and adverse drug reactions. Can Med Assoc | 1999; 160:987-988.

7. Mittmann N, Knowles SR, Gomez M, Fish JS, Cartotto R, Shear NH. Evaluation of the extent of under-reporting of serious adverse drug reactions: the case of toxic epidermal necrolysis. Drug Saf 2004; 27:477-487.

Evidence-Based Dentistry (2005) 6, 67.

doi:10.1038/sj.ebd.6400340 